\title{
The impact of variations in care and complications within a colorectal Enhanced Recovery After Surgery program on length of stay
}

\author{
James Wei Tatt Toh ${ }^{1,2,3}$, Jack Cecire ${ }^{1}$, Kerry Hitos ${ }^{1,2,4}$, Karen Shedden ${ }^{2}$, Fiona Gavegan ${ }^{2}$, \\ Nimalan Pathmanathan ${ }^{1,2}$, Toufic El Khoury ${ }^{1,2,5}$, Angelina Di Re ${ }^{2}$, Annelise Cocco ${ }^{2}$, Alex Limmer ${ }^{2}$, \\ Tom Liang ${ }^{2}$, Kar Yin Fok ${ }^{2}$, James Rogers ${ }^{2}$, Edgardo Solis ${ }^{2}$, Grahame Ctercteko ${ }^{1,2}$ \\ ${ }^{1}$ Discipline of Surgery, Sydney Medical School, The University of Sydney, Sydney, ${ }^{2}$ Department of Surgery, Westmead Hospital, Westmead, \\ ${ }^{3}$ Discipline of Surgery, University of New South Wales, Sydney, ${ }^{4}$ Westmead Research Centre for Evaluation of Surgical Outcomes, Westmead \\ Hospital, Sydney, ${ }^{5}$ Discipline of Medicine, University of Notre Dame Australia, Sydney, NSW, Australia
}

Purpose: Enhanced Recovery After Surgery (ERAS) has become standard of care in colorectal surgery. However, there is not a universally accepted colorectal ERAS protocol and significant variations in care exist between institutions. The aim of this study was to examine the impact of variations in ERAS interventions and complications on length of stay (LOS). Methods: This study was a single-center review of the first 200 consecutive patients recruited into our prospectively collected ERAS database. The primary outcome of this study was to examine the rate of compliance to ERAS interventions and the impact of these interventions on LOS. The secondary outcome was to assess the impact of complications (anastomotic leak, ileus, and surgical site infections) on LOS. ERAS interventions, rate of adherence, LOS, readmissions, morbidity, and mortality were recorded, and statistical analysis was performed.

Results: ERAS variations and complications significantly influenced patient LOS on both univariate and multivariate analysis. ERAS interventions identified as the most important strategies in reducing LOS included laparoscopic surgery, mobilization twice daily postoperative day (POD) 0 to 1 , discontinuation of intravenous fluids on POD 0 to 1 , upgrading to solid diet by POD 0 to 2, removal of indwelling catheter by POD 0 to 2, avoiding nasogastric tube reinsertion and removing drains early. Both major and minor complications increased LOS. Anastomotic leak and ileus were associated with the greatest increase in LOS.

Conclusion: Seven high-yield ERAS interventions reduced LOS. Major and minor complications increased LOS. Reducing variations in care and complications can improve outcomes following colorectal surgery.

\section{Keywords: Enhanced Recovery After Surgery; Colorectal surgery; Colonic neoplasms; Rectal neoplasms}

\section{INTRODUCTION}

Enhanced Recovery After Surgery (ERAS) protocols have become

Received: Sep 8, 2020 - Revised: Nov 22, 2020 - Accepted: Nov 23, 2020 Correspondence to: James Wei Tatt Toh, BSc, MBBS (UNSW), FRACS Department of Surgery, Westmead Hospital, Cnr Hawkesbury and Darcy Rd, Westmead, NSW 2145, Australia

Tel: +61-288905555, Fax: +61-280111276

E-mail: james.toh@health.nsw.gov.au

ORCID: https://orcid.org/0000-0002-0110-2629

(C) 2022 The Korean Society of Coloproctology

This is an open-access article distributed under the terms of the Creative Commons Attribution NonCommercial License (https://creativecommons.org/licenses/by-nc/4.0) which permits unrestricted noncommercial use, distribution, and reproduction in any medium, provided the original work is properly cited. standard practice in colorectal surgery since it was first introduced over 20 years ago. ERAS was first described in 1997 by Kehlet [1]. Six factors were identified; preoperative information and teaching, attenuation of stress, pain relief, exercise, enteral nutrition, and growth factors. In 2005, Fearon et al. [2] published a consensus of 17 main elements of the ERAS protocol. In 2017, Ljungqvist et al. [3] updated the ERAS consensus, dividing it into 4 categories (preadmission, preoperative, intraoperative, and postoperative). Preadmission nutritional support, cessation of smoking and control of alcohol intake, anesthetic medical optimization and preoperative counseling; preoperative selective bowel preparation, carbohydrate loading, no nil by mouth and postoperative nausea \& vomiting prophylaxis; intraoperative minimally 
invasive surgery, minimization of drains and tubes, regional analgesia, opioid-sparing anesthesia, balanced fluids, and temperature control; postoperative early removal of drains and tubes, stopping intravenous (IV) fluids, multimodal opioid-sparing pain control, early mobilization, early oral intake of fluids and solids, post discharge follow-up [3].

While most institutions have adopted the ERAS principle of optimizing patient care pre-, intra-, and postoperatively to improve outcomes and reduce the length of stay (LOS), there have still been significant variations in care and outcomes between colorectal units, and even between colorectal surgeons. There may be several reasons for this. Firstly, ERAS principles are not specific to colorectal surgery, but rather principles to improve postoperative outcomes in general. This has led to an approach to ERAS where institutions prioritize specific ERAS principles into their existing programs of care rather than implementing all of the principles. Secondly, ERAS interventions are guided by a set of principles and must be guided by the best available evidence in the literature. Of the 17 ERAS interventions described in 2005 by Fearon et al. [2], 2 of the interventions have been challenged by level 1 evidence. Several meta-analyses have shown that while nonsteroidal anti-inflammatory drugs (NSAIDs) may improve ileus rates, NSAIDs may lead to increased risk of anastomotic leak [4-7]. Similarly, several meta-analyses and a network meta-analysis have demonstrated that avoidance of bowel preparation is associated with increased rates of infectious complications postoperatively, and that mechanical bowel preparation with oral antibiotics was associated with better outcomes [8-11]. Studies using international databases such as the National Surgical Quality Improvement Program (NSQIP) [12] have reported that avoidance of bowel preparation may be associated with increased risk of anastomotic leak following left-sided restorative procedures [13]. Of note, the ERAS society has recently changed its recommendation of avoiding bowel preparation to selective bowel preparation. Finally, variations in care and outcomes may be due to differences in adherence to ERAS strategies and deficiencies in audit of compliance and outcomes.

In this study, we studied the variations in care and complications for the first 200 consecutive patients recruited into our prospective ERAS database at our institution.

\section{METHODS}

\section{Patients and data collection}

A prospectively collected database divided into 4 sections consisting of 55 data points $(11,33,1$, and 10) was created on the Research Electronic Data Capture (REDCap) data management platform $[14,15]$. The REDCap data management platform was developed in 2004 at Vanderbilt University and has since been used as a valid database management platform by research teams. The database was de-identified, with each patient assigned a patient identification (ID) number. Institutional Review Board ap- proval for this study was granted (No. 5878-AU RED LNR/18/ WMEAD/424).

Consecutive patients from mid-2017 to 2018 suitable for ERAS were identified at the preadmission clinic by ERAS nurses. All elective major colorectal surgery were included. Emergencies, minor procedures, and palliative procedures and cases associated with bowel obstruction were excluded. Of the 200 patients included, 29 were excluded from the analysis. These patients were excluded from analysis as they were emergency cases, reversal of stoma, laparoscopic appendicectomy only, transanal endoscopic microsurgery/transanal minimally invasive surgery, did not proceed with surgical procedure, or incorrectly identified for ERAS.

The ERAS interventions included in our ERAS pathway between 2017 and 2018 and the updated pathway from 2019 have been included in Supplementary Figs. 1, 2. Where appropriate, the ERAS nurses generated a patient ID and completed the first section consisting of details on patient demographic, preadmission and preoperative ERAS interventions at the preadmission clinic. Once the ID has been generated, junior medical officers, registrars, and fellows on the colorectal unit were prompted to complete the second section which evaluated intraoperative and postoperative care including ERAS interventions. Issues with the data were raised and clarified at regular ERAS meeting every 2 to 4 weeks attended by a specialist consultant colorectal surgeon with the medical officers and nurses on the colorectal unit. At these meetings, complications, readmissions, and mortality were discussed within the group and checked via the hospital Electronic Medical Record Powerchart. On reaching consensus, complications based on Clavien-Dindo classification (CD) $[16,17]$ (section 3) as well as 8 complications (superficial, deep, organ/space occupying surgical site infection [SSI], anastomotic leak, urinary tract infection [UTI], pneumonia, deep venous thrombosis/pulmonary embolism (DVT/PE), prolonged ileus (POI) as well as readmission within 30 days and death within 30 days were recorded by the colorectal consultant supervising the ERAS database. The REDCap ERAS database was then linked to the NSQIP data which at the time of linkage collected 176 data points.

The primary outcome of this study was to examine the rate of compliance to ERAS interventions and the impact of these interventions on LOS. The secondary outcome was to assess the impact of complications based on CD (minor, CD I/II; major, CD III/IV) and type of complication (anastomotic leak, ileus, and SSIs) on LOS.

\section{Statistical analysis}

Data on ERAS interventions, complications, and LOS were analyzed using IBM SPSS Statistics ver. 26.0 for Windows (IBM Corp., Armonk, NY, USA) and STATA (Stata/MP, ver. 15; StataCorp LLC, College Station, TX, USA). The normality of data distribution was evaluated and summarized using descriptive statistics. Continuous data were presented by means with their standard deviations or medians and interquartile ranges (IQR). The 
chi-square test or Mann-Whitney U-tests was used to test for differences.

With ERAS interventions and complications as predictors, LOS was analyzed both as a continuous outcome variable as well as converted to a binary outcome based on median ( $\leq 6$ and $>6$ days). Subsequently, both a univariate and multivariate linear and logistic regression analysis was performed respectively. Where there were more than 2 categories for the independent variable, a 1-way analysis of variance was performed instead of univariate linear regression. All tests were 2-tailed and a P-value of $<0.05$ was considered significant.

\section{RESULTS}

\section{Study population}

In total, 171 patients were suitable for inclusion for analysis. Table 1 shows patient demographics, indications for surgery, and procedural characteristics. Ninety-nine patients $(57.9 \%)$ were male. The majority of patients (81.3\%) had cancer, $7.6 \%$ had diverticulitis, and $1.2 \%$ had Crohn disease; there were no patients with volvulus and 8.2\% had other indications. The median age was 69.1 years (IQR, 57.7-66.6 years). In terms of preanesthetic comorbidities, the American Society of Anesthesiologist (ASA) physical status (PS) classification system was used. The majority of patients were ASA PS grade I or II (healthy or mild systemic disease; $54.4 \%$ ) or ASA PS grade III (severe systemic disease; $40.9 \%$ ). There were no patients classified as ASA PS grade V (moribund patient not expected to survive) or VI (brain-dead). The median body mass index (BMI) was $27.8 \mathrm{~kg} / \mathrm{m}^{2}$ (overweight). The most common procedure performed was a right hemicolectomy (33.3\%) followed by high anterior resection (26.3\%) and low or ultra-low anterior resection (22.8\%). The rates of nonrestorative proctectomies were low (Hartmann's procedure, 1.8\%; abdominoperineal resection, $0.6 \%$ ). Univariate analysis of LOS based on patient, disease, and surgical characteristics have been provided in Supplementary Table 1, and these were included for assessment in fitting the multivariable model (see Discussion).

Compliance with and impact of ERAS interventions on LOS Compliance with ERAS interventions within our cohort is detailed in Table 2. There was good compliance with preadmission counseling (100\%), carbohydrate loading (97.1\%), and preoperative bowel preparation (91.2\%) (Fig. 1). During the period of this study, immunonutrition and preoperative oral antibiotics had not been introduced ( $0 \%$ and $1.2 \%$, respectively). Approximately $70 \%$ of patients had liquids up to 3 hours prior to surgery, and $6.3 \%$ of patients required preoperative iron transfusion for anemia. It is not known how many patients had iron deficiency anemia but did not receive iron transfusion.

Intraoperatively, the majority of operations were performed laparoscopically. Robotic surgical platforms were not available during the study period. Of the operations, $74.3 \%$ were commenced
Table 1. Patient demographics, indications for surgery, and procedural characteristics $(\mathrm{n}=171)$

\begin{tabular}{lc}
\hline Variable & Data $^{\mathrm{a}}$ \\
\hline Age (yr) & $69.1(57.7-66.6)$ \\
Female sex & 42.1 \\
ASA PS classification & \\
I & 8.8 \\
॥ & 45.6 \\
III & 40.9 \\
Body mass index $\left(\mathrm{kg} / \mathrm{m}^{2}\right)$ & $27.8(24.2-31.7)$ \\
HbA1c (\%) & $5.6(5.3-6.3)$ \\
Indication for surgery & \\
Cancer & 81.3 \\
Crohn disease & 1.2 \\
Ulcerative colitis & 0.5 \\
Diverticulitis & 7.6 \\
Recurrent volvulus & 0 \\
Others & 8.2
\end{tabular}

Procedure

$\begin{array}{lr}\text { Right hemicolectomy } & 33.3 \\ \text { Transverse colectomy } & 0.6 \\ \text { Left hemicolectomy } & 2.3 \\ \text { High anterior resection } & 26.3 \\ \text { Low anterior resection } & 12.3 \\ \text { Ultra-low anterior resection } & 10.5 \\ \text { Anterior resection unspecified } & 4.1 \\ \text { Hartmann's procedure } & 1.8 \\ \text { Abdominoperineal resection } & 0.6 \\ \text { Total/subtotal colectomy } & 1.2 \\ \text { Total proctocolectomy } \pm \text { IPAA } & 1.8 \\ \text { Others } & 5.3\end{array}$

Values are presented as median (interquartile range) or percentage. ASA, American Society of Anesthesiologist; PS, physical status; HbA1c, glycosylated hemoglobin A1c; IPAA, ileal pouch anal anastomosis.

${ }^{a}$ Does not include missing data (minimal); see Supplementary Table 2.

by minimally invasive laparoscopic surgery with $11.1 \%$ subsequently requiring conversion to open surgery. Fourteen percent of cases were hybrid, where both laparoscopic and open surgery were performed (i.e., where the splenic flexure was mobilized laparoscopically, and rectal dissection was performed open). Handassisted operations were also considered hybrid.

Postoperatively, there was good compliance with multimodal pain management (88.2\%). Patients of $82.9 \%$ were mobilized twice daily by postoperative day (POD) 1 and $85.4 \%$ were prescribed postoperative antiemetics.

However, there were also ERAS interventions with a lower 
Table 2. Compliance with ERAS interventions $(n=171)$

\begin{tabular}{|c|c|}
\hline ERAS intervention & Agreement rate $(\%)^{\mathrm{a}}$ \\
\hline Preadmission counseling & 100 \\
\hline Carbohydrate loading & 97.1 \\
\hline Immunonutrition & 0 \\
\hline \multicolumn{2}{|l|}{ Surgical access } \\
\hline Open & 11.7 \\
\hline Laparoscopic & 63.2 \\
\hline Laparoscopic converted to open & 11.1 \\
\hline Hybrid & 14 \\
\hline Bowel preparation & 91.2 \\
\hline Oral antibiotics & 1.2 \\
\hline Liquids up to $3 \mathrm{hr}$ prior to surgery & 73.7 \\
\hline Epidural & 5.3 \\
\hline Multimodal pain management & 88.2 \\
\hline NSAIDs & 31.8 \\
\hline Preoperative iron transfusion for anemia & 6.3 \\
\hline Postoperative antiemetics & 85.4 \\
\hline Mobilization POD 0-1 & 83 \\
\hline Mobilization BD POD 0-1 & 82.9 \\
\hline IV fluids discontinued POD 0-1 & 40.1 \\
\hline Upgraded to solid diet POD 0-2 & 71.4 \\
\hline \multicolumn{2}{|l|}{ IDC } \\
\hline IDC out POD 0-2 & 79.5 \\
\hline IDC out $P O D \geq 3$ & 20.5 \\
\hline Failed TOV, IDC reinsertion required & 8.8 \\
\hline \multicolumn{2}{|l|}{ NGT } \\
\hline NGT out POD 0-2 & 3.5 \\
\hline NGT out POD $\geq 3$ & 8.8 \\
\hline No NGT & 87.7 \\
\hline NGT reinsertion & 14.6 \\
\hline \multicolumn{2}{|l|}{ Drain } \\
\hline No drain & 54.7 \\
\hline Drain removed POD 0-2 & 10.6 \\
\hline Drain removed POD 3-4 & 17.6 \\
\hline Drain removed POD $\geq 5$ & 14.6 \\
\hline
\end{tabular}

ERAS, Enhanced Recovery After Surgery; NSAIDs, nonsteroidal anti-inflammatory drugs; POD, postoperative day; BD, twice a day; IV, intravenous; IDC, indwelling catheter; TOV, trial of void; NGT, nasogastric tube.

aDoes not include missing data (minimal); see Supplementary Table 3.

agreement rate. $28.6 \%$ of patients had not started solid diet by POD 2. $20.5 \%$ of patients had their indwelling catheter (IDC) removed POD $\geq 3$ and $8.8 \%$ had their nasogastric tube (NGT) removed POD $\geq 3$. The IDC and NGT reinsertion rate were $8.8 \%$ and $14.6 \%$, respectively. Few patients had epidurals (5.3\%). NSAIDs were prescribed in $31.8 \%$ of cases. While approximately half the patients did not have a drain, $14.6 \%$ had drains removed $\mathrm{POD} \geq 5$.

Of the 18 ERAS intervention (preoperative 7, intraoperative 2, and postoperative 9), this study identified 7 ERAS interventions that influenced LOS on univariate linear regression, of which 4 maintained a statistically significant difference on multivariate analysis. On univariate logistic regression analysis, 10 ERAS interventions influenced LOS (Table 3), of which 5 remained statistically significant on multivariate analysis. Several of the preoperative ERAS interventions were not estimable as there was a close to $100 \%$ agreement rate.

On univariate linear regression analysis, laparoscopic surgery when compared to open (7.0 [5.1-9.0] vs. 10.8 (6.3-15.3), $\mathrm{P}=$ 0.068 ), mobilization twice a day (bis die, BD) on POD 0 to 1 (7.6 [5.9-9.3] vs. 13.0 [9.1-16.9], $\mathrm{P}=0.013)$, discontinuation of IV fluids on POD 0 to 1 (5.9 [3.5-8.4] vs. 10.4 [8.4-12.4], $\mathrm{P}=0.006)$, upgrading to solid diet by POD 0 to $2(6.3$ [4.6-8.1] vs. 14.2 [11.5-17.0], $\mathrm{P}<0.0001)$, removing the IDC by POD 0 to $2(7.6$ [5.9-9.3] vs. 12.7 [9.3-16.2], $\mathrm{P}=0.009)$, avoiding NGT reinsertion (7.2 [5.6-8.8] vs. 16.8 [12.9-20.7], $\mathrm{P}<0.0001)$, and removing drains early $(\mathrm{P}<0.0001)$ was associated with a statistically significant reduction or trend in reduction of LOS. On multivariate linear regression analysis, laparoscopic surgery (when compared with patients who had open surgery with epidurals), upgrading to solid diet by POD 0 to 2, avoiding NGT reinsertion and removing drains prior to POD 4 were associated with a statistically significant reduction in LOS.

On univariate logistic regression analysis ( $\leq 6$ and $>6$ days), laparoscopic surgery $(\mathrm{P}=0.024)$, multimodal pain management (odds ratio [OR], 4.48; 95\% confidence interval [CI], 1.25-16.02; $\mathrm{P}=0.021$ ), patient controlled analgesia (PCA) duration $(\mathrm{OR}, 1.63$; 95\% CI, 1.22-2.17; $\mathrm{P}=0.001$ ), BD mobilization (OR, 0.28; 95\% CI, 0.11-0.71; $\mathrm{P}=0.007)$, IV fluid duration (OR, 2.21; 95\% CI, $1.60-3.07$; $<<0.0001)$, time to solid diet (OR, 0.16; 95\% CI, 0.080.34; $\mathrm{P}<0.0001$ ), IDC duration $\mathrm{POD} \geq 3$ (OR, 5.37; 95\% CI, 2.32-12.46; $\mathrm{P}<0.0001)$, NGT reinsertion (OR, 46.48; 95\% CI, 6.09-354.53; $\mathrm{P}<0.0001)$, drain placement $(\mathrm{P}=0.005)$, and time to removal POD $\geq 5$ (OR, 5.34; 95\% CI, 2.12-13.45, P < 0.0001) impacted on LOS. On multivariate logistic regression analysis, epidural, BD mobilization, time to solid diet, IDC duration, and NGT duration were factors associated with LOS.

\section{Impact of complications on LOS}

Overall, $56.1 \%$ of patients did not have any complications. Of those who had complications, $33.9 \%$ of patients had minor (CD III) complications. Only $8.2 \%$ of patients had major (CD III-IV) complications and the mortality rate was $1.2 \%$. The most common complication was ileus (13.5\%). The overall SSI rate was $11.1 \%$ and the leak rate was $4.7 \%$. The rate of UTI, pneumonia, and DVT/PE were 5.3\%, 2.9\%, and 1.2\%, respectively (Table 4, Fig. 2). 


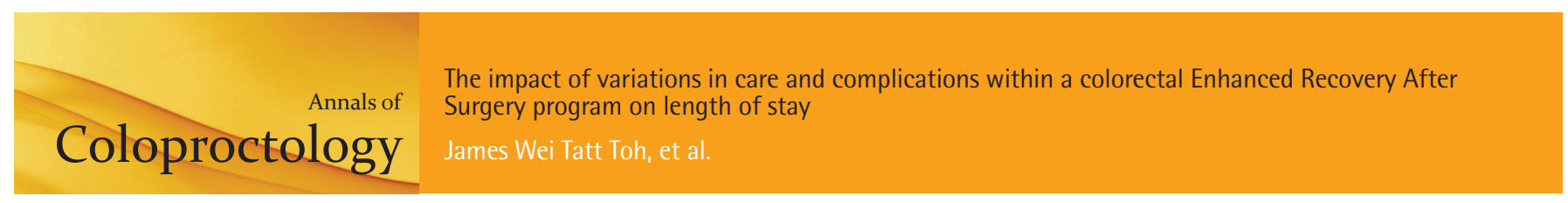

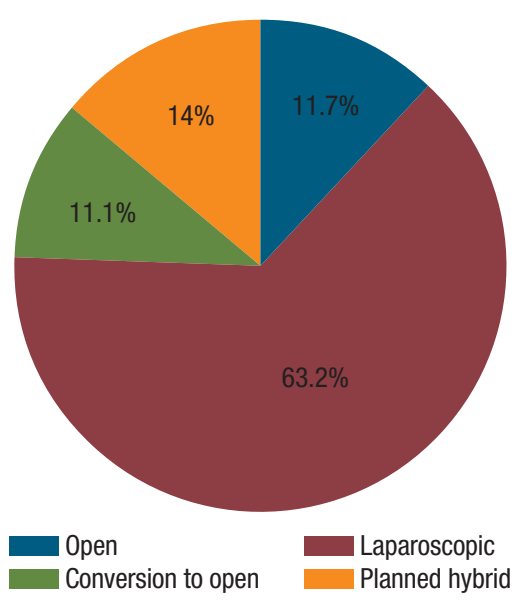

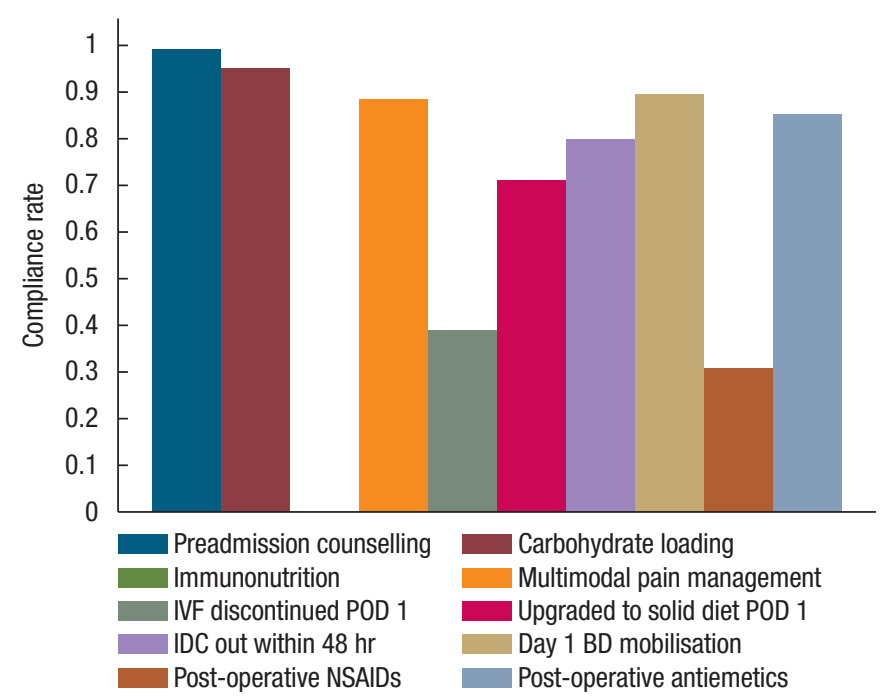

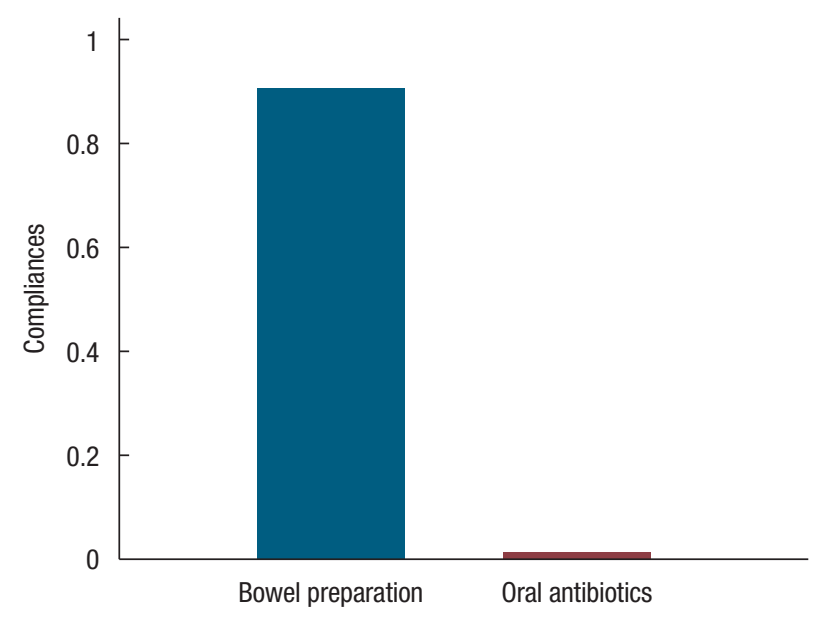
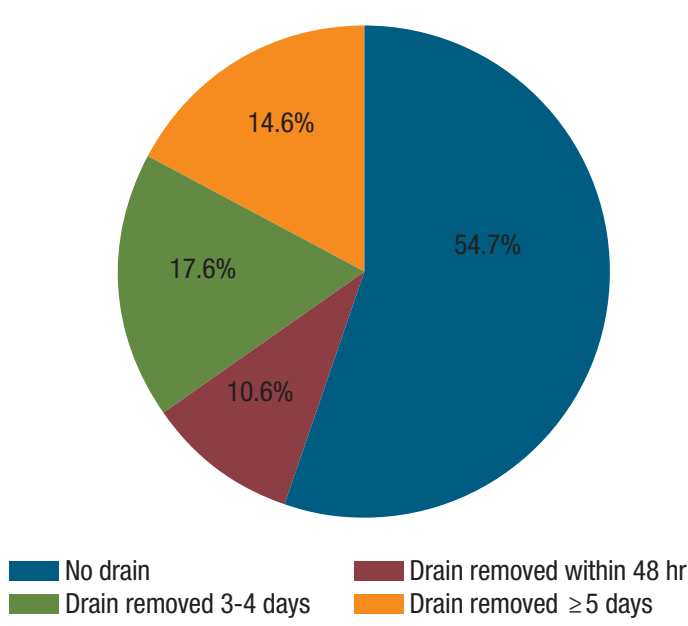

Fig. 1. Compliance with Enhanced Recovery After Surgery (ERAS) interventions. POD, post-operative day; IDC, indwelling catheter; NSAIDs, non-steroidal anti-inflammatory drugs; BD, twice a day.

Complications had a significant impact on LOS on both linear regression $(\mathrm{P}<0.0001)$ and logistic regression $(\mathrm{P}<0.0001)$ (Table 5). The mean LOS without complication was 4.9 days (4.5-5.3 days). For patients who had CD I, II, III, and IV complications, the LOS was 8.2 days (6.5-9.9 days), 12.9 days (8.4-17.3 days), 14.4 days (6.9-21.9 days), and 38.0 days (8.8-67.2 days), respectively. Importantly, both minor (CD I/II) and major (CD III/IV) complications increased the risk of LOS $\geq 6$ days. For CD I, the OR was 2.88 (95\% CI, 1.19-6.96; $\mathrm{P}=0.019)$; and for CD II, the OR was 6.11 (95\% CI, 2.56-14.62; $\mathrm{P}<0.0001)$.

Anastomotic leak increased LOS by 23 days (31.0 [8.8-53.2] vs. 7.5 [6.3-8.6], $\mathrm{P}<0.0001)$. Ileus increased LOS by 13 days ( 20.2 [12.9-27.5] vs. 6.7 [5.5-7.9], $\mathrm{P}<0.0001)$ (Fig. 3). SSI increased LOS by 7 days (15.4 [7.9-23] vs. 8.0 [6.5-9.6], $\mathrm{P}=0.016$ ). UTI also significantly increased LOS, but it was usually associated with other complications and its effect on LOS was complex. Organ/ space SSI shared a similar complication profile to anastomotic leak both by definition and its effect on LOS (24.3 [14.0-34.8] vs. 8.1 [6.6-9.6], $\mathrm{P}<0.0001$ ) (Table 5).

\section{DISCUSSION}

This retrospective review of a prospectively collected database has provided an insight into the preoperative, intraoperative, and postoperative variables including patient demographics, disease state, and surgical technique that may influence the outcomes achieved in a colorectal ERAS program. Of the 18 ERAS intervention, the most important predictors of decreased LOS were laparoscopic surgery, mobilization BD POD 0 to 1 , discontinuation of IV fluids on POD 0 to 1 , upgrading to solid diet by POD 0 to 2, removing the IDC by POD 0 to 2, avoiding NGT reinsertion and removing drains early. Several of these interventions were 
Table 3. Univariate linear and logistic regression of ERAS interventions and hospital length of stay (LOS)

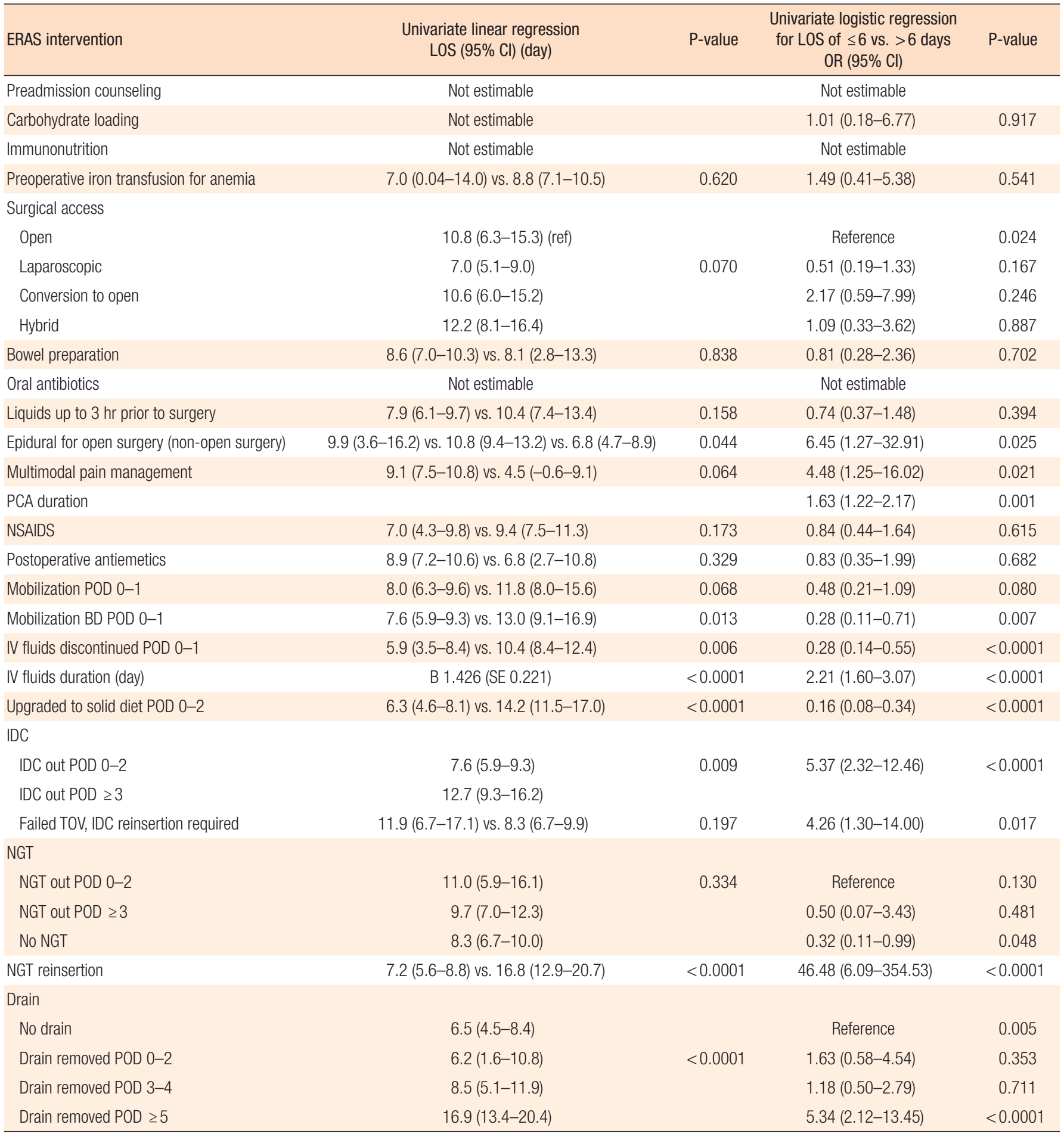

ERAS, Enhanced Recovery After Surgery; Cl, confidence interval; OR, odds ratio; PCA, patient controlled analgesia; NSAIDs, nonsteroidal anti-inflammatory drugs; POD, postoperative day; BD, twice a day; IV, intravenous; IDC, indwelling catheter; TOV, trial of void; NGT, nasogastric tube.

also statistically significant on multivariate analysis including laparoscopic surgery (when compared with patients who had open surgery with epidurals), upgrading to solid diet by POD 0 to 2, avoiding NGT reinsertion, and removing drains prior to POD 4. 
Table 4. Postoperative length of stay, morbidity, mortality, and readmissions $(\mathrm{n}=171)$

\begin{tabular}{lc}
\hline Variable & Data $(\%)^{\mathrm{a}}$ \\
\hline Length of stay (day) & \\
Median (interquartile range) & $6(4-9)$ \\
Mean \pm standard deviation & $8.56 \pm 10.22$ \\
Clavien-Dindo classification & \\
No complications & 56.1 \\
I & 14.6 \\
II & 19.3 \\
III & 4.7 \\
IV & 3.5 \\
V & 1.2 \\
Complication & \\
Superficial SSI & 7.0 \\
Deep SSI & 1.2 \\
Organ space SSI & 2.9 \\
Anastomotic leak & 4.7 \\
Urinary tract infection & 5.3 \\
Pneumonia & 2.9 \\
DVT/PE & 1.2 \\
Ileus & 13.5 \\
Readmission within 30 days & 7.6 \\
Death within 30 days & 1.2 \\
\hline SSI & \\
\hline
\end{tabular}

SSI, surgical site infection; DVT, deep vein thrombosis; PE, pulmonary embolism. aNo data missing.

When we analyzed the data based on a binary outcome (LOS of $\leq 6$ and $>6$ days), laparoscopic surgery, multimodal pain management, PCA duration, BD mobilization, IV fluid duration, epidural, time to solid diet, IDC duration, avoidance of NGT placement and NGT reinsertion, drain placement and time to removal were associated with LOS $\leq 6$ days. On multivariate analysis, epidural, BD mobilization, time to solid diet, IDC duration, and NGT duration were factors associated with LOS.

There was a low rate of perioperative mortality and major complications. However, there was a higher rate of minor complications (CD I/II) than expected. This, in part, corresponded to the patients who had POI, UTI, and SSI. The rate of POI in our cohort was $13.5 \%$, total SSI was $11.1 \%$, and UTI rate was $5.3 \%$. The Binational Colorectal Cancer Audit (BCCA) database reported a lower ileus rate of $8 \%$ and a wound infection rate of $4 \%$; the rate of UTI was not reported [18]. We used the international consensus definition of POI in our study. POI was defined as 2 or more of the following on or after day 4 ; nausea or vomiting, inability to tolerate oral diet over 24 hours, no flatus over 24 hours, abdominal distension; and radiological confirmation of ileus.

In this study, ileus increased LOS by approximately 13 days, SSI

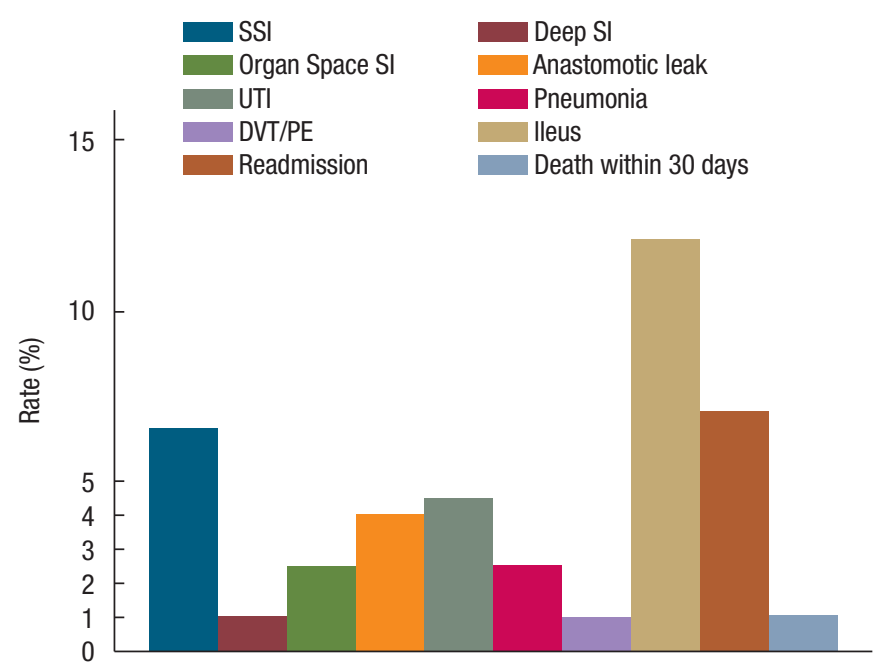

Fig. 2. Rate of postoperative complications, readmissions and mortality. SSI, surgical site infection; UTI, urinary tract infection; DVT, deep venous thrombosis; PE, pulmonary embolism.

increased LOS by 7 days, UTI increased LOS by 11 days, and anastomotic leak increased LOS by 23 days. These results are similar to the study by Iversen et al. [19] which reported an increased LOS by 16 days with anastomotic leak as well as the study by Lluis and Biondo [20] which reported an increased LOS by 9 days with ileus.

Based on the low leak rate, low major complication rate, and the LOS, this indicated that the quality of surgery and postoperative management was satisfactory. In this study, the leak rate (4.7\%) and LOS (8.56 days) were comparable to the results reported in the BCCA (2017) for Australia and New Zealand. In this report, the anastomotic leak rate was $4 \%$, mean LOS was 8.5 days, and the surgical complication rate was $31.1 \%$ [18]. While the ALCCaS (Australasian Laparoscopic Colon Cancer Study) trial reported a lower anastomotic leak rate as $1.4 \%$ for laparoscopic and $3.4 \%$ for open [21], overall, the MRC CLASICC (Medical Research Council Conventional versus Laparoscopic-Assisted Surgery in Colorectal Cancer) trial (anastomotic leak rate for colon surgery as 3\% [open and laparoscopic], 7\% [open], and 10\% [laparoscopic] for rectal surgery) [22], the 2010 Dutch Surgical Colorectal Audit (anastomotic leak of $8.7 \%$ after restorative colorectal surgery $[n=9,192]$ in the Netherlands) [23], and the ALaCaRT (Australasian Laparoscopic Cancer of the Rectum Trial) randomized clinical trial (anastomotic leak rate following rectal cancer surgery, 7\% [3\% for grade 3/4 leaks]) [24] reported higher anastomotic leak rates.

The rate of conversion to open in this study was $11.1 \%$. This was better than the rate of conversion to open reported in the MRC CLASICC trial (colon and rectal surgery, 29\%) [22] and ALCCaS (colon surgery, 14.6\%) [21], but slightly higher than the recent ALaCaRT (rectal surgery, 9\%) [24] and the BCCA [18].

We included patients with a median BMI of $27.8 \mathrm{~kg} / \mathrm{m}^{2}$ (IQR, 
Table 5. Univariate linear and logistic regression of complications and hospital length of stay

\begin{tabular}{|c|c|c|c|c|}
\hline Variable & Univariate linear regression & P-value & Logistic regression & P-value \\
\hline \multicolumn{5}{|l|}{ Clavien-Dindo classification } \\
\hline No complications & $4.9(4.5-5.3)$ & & Reference & $<0.0001$ \\
\hline I & $8.2(6.5-9.9)$ & $<0.0001$ & $2.88(1.19-6.96)$ & 0.019 \\
\hline$\|$ & $12.9(8.4-17.3)$ & & $6.11(2.56-14.62)$ & $<0.0001$ \\
\hline III & $14.4(6.9-21.9)$ & & $4.43(0.87-22.64)$ & 0.074 \\
\hline IV & $38.0(8.8-67.2)$ & & $7.35(0.84-64.34)$ & 0.072 \\
\hline V & No estimate & & No estimate & No estimate \\
\hline \multicolumn{5}{|l|}{ Type of complication } \\
\hline Superficial SSI & 15.4 (7.9-23.0) vs. 8.0 (6.5-9.6) & 0.016 & $2.98(0.86-10.33)$ & 0.084 \\
\hline Deep SSI & No estimate & No estimate & No estimate & No estimate \\
\hline Organ space SSI & $24.3(14.0-34.8)$ vs. 8.1 (6.6-9.6) & $<0.0001$ & $2.12(0.35-13.02)$ & 0.418 \\
\hline Anastomotic leak & 31.0 (8.8-53.2) vs. 7.5 (6.3-8.6) & $<0.0001$ & No estimate & No estimate \\
\hline Urinary tract infection & 19.1 (4.7-33.6) vs. 8.0 (6.5-9.4) & 0.001 & $12.32(1.50-100.88)$ & 0.019 \\
\hline Pneumonia & 20.8 (1.9-39.7) vs. 8.2 (6.7-9.7) & 0.006 & No estimate & No estimate \\
\hline Deep venous thrombosis/pulmonary embolism & No estimate & No estimate & No estimate & No estimate \\
\hline lleus & 20.2 (12.9-27.5) vs. 6.7 (5.5-7.9) & $<0.0001$ & No estimate & No estimate \\
\hline Readmission within 30 days & $8.9(5.7-12.1)$ vs. $8.6(6.9-10.3)$ & 0.908 & $2.34(0.73-7.47)$ & 0.152 \\
\hline Death within 30 days & No estimate & No estimate & No estimate & No estimate \\
\hline
\end{tabular}

Values are presented as days (95\% confidence inteval [Cl]) for linear regression and odds ratio ( $95 \% \mathrm{Cl}$ ) for logistic regression.

SSI, surgical site infection.

$24.2-31.7 \mathrm{~kg} / \mathrm{m}^{2}$ ) which is high and a typical reflection of the patient population in our local health district. BMI was not shown to be a significant predictor of LOS or major complications.

The median LOS for our ERAS cohort was 6 days. At the same institution, prior to commencement of ERAS, the median LOS for the unit was 8 days (no nonmedical reason for delay) to 15 days (medical reason for delay) [25]. Since the commencement of ERAS, the overall median LOS of 6 days demonstrated a significant improvement in outcome (Table 6).

Consistently, in this study, the most important ERAS interventions associated with of decreased LOS on all analysis were laparoscopic surgery, BD mobilization from POD 0 to 1, early discontinuation of IV fluids by POD 0 to 1 , upgrading to solid diet by POD 2, removal of IDC by POD 2, avoidance of NGT placement and reinsertion and removal of drain by POD 4.

Other ERAS studies have identified avoidance of oral opiates [26], duration of use of epidurals [26], age [27], complications [27], nasogastric and IDC reinsertion, continued IV fluid infusion and inability to mobilize [28], and reduced compliance to ERAS protocols [29] to be associated with increased LOS.

The majority of deviations from the ERAS pathway were in the postoperative period. While lack of agreement or noncompliance was due to medical necessity in a majority of cases, approximately $30 \%$ of deviations could have been improved. This was a similar finding to the study by Roulin et al. [30] $(n=76)$.
While our median LOS is better than the median LOS reported in the BCCA and several randomized controlled trials and equivalent to the LOS reported in NSQIP studies, several ERAS programs have reported median LOS of between 3 and 5 days [3134]. Future studies are required to evaluate which patients can be further fast-tracked safely without increasing readmission rates and morbidity.

The emphasis of this study was quality of data with team and specialist consensus of patient outcomes collected prospectively at ERAS meetings with strict criteria for outcome assessments. This study has driven several quality improvement programs at our institution including an SSI reduction bundle (SSIRB), a project to reduce ileus and UTIs. Our SSIRB consists of bowel preparation and oral antibiotics $[8,13]$, IMPACT (Nestle, Vevey, Switzerland) immunonutrition, the use of chlorhexidine and alcohol preparation, wound protectors, separate closing tray and change of gloves prior to closure, and avoidance of nonselective NSAIDs to reduce the risk of anastomotic leak. Our ileus project consists of reducing opiate use and optimizing multimodal analgesia and decreasing the duration of PCA. To reduce UTIs, IDC removal as early as medically possible has been prioritized. It is anticipated that improvements in outcomes achieved by successful ERAS and quality improvement programs will be associated with cost savings [3234].

This study has the limitations as follows. Due to the size of the 

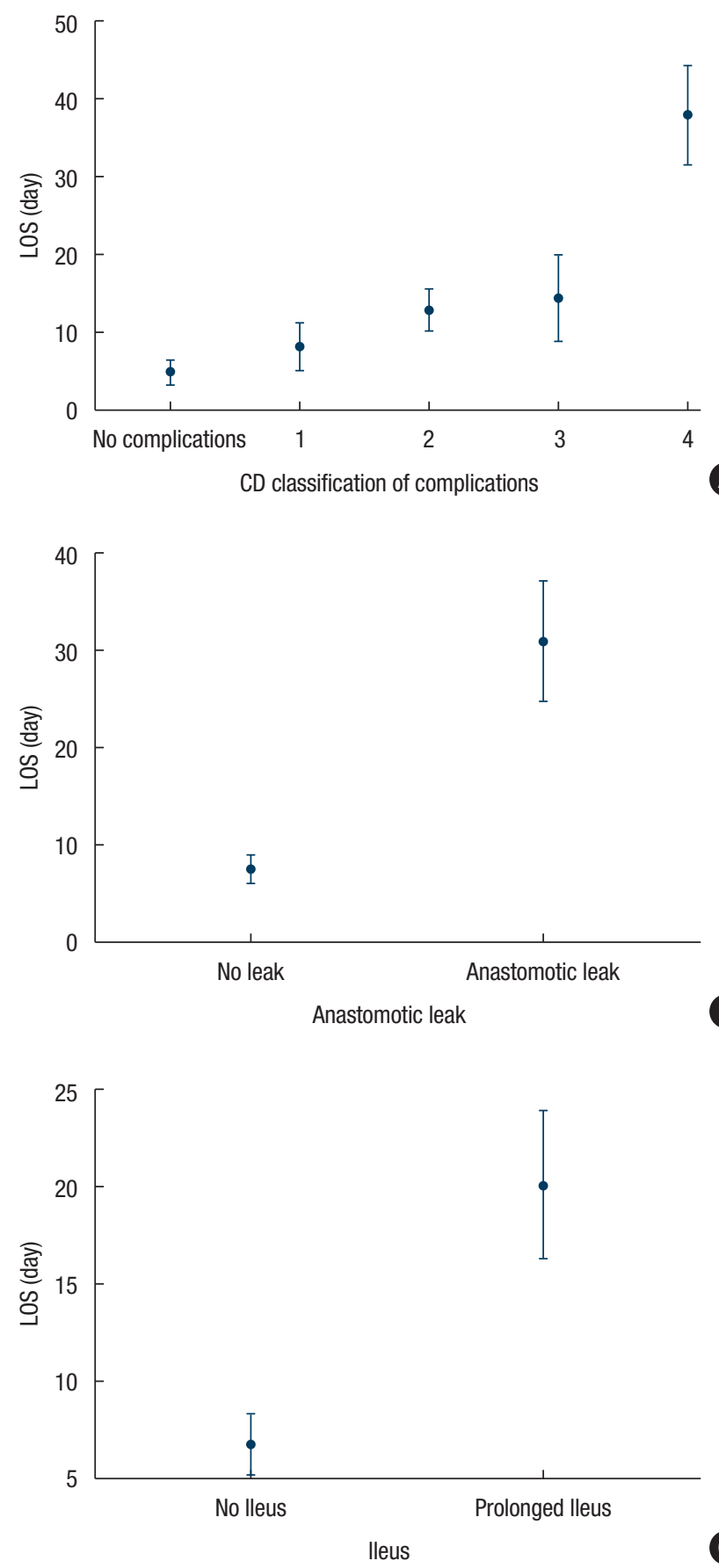

Fig. 3. The association between complications (by $\mathrm{CD}$, anastomotic leak and ileus) and length of stay. (A) Complications by CD score and LOS, (B) Prolonged ileus and LOS, (C) Anastomotic leak and LOS.

CD, Clavien-Dindo; LOS, length of stay.

study, many factor variables fell out of the multivariable model $(\mathrm{P}>0.1)$ despite displaying a trend to significance, influencing the multivariate statistical model. For this reason, we have included
Table 6. Comparison of postoperative length of stay, readmission, and mortality pre-ERAS and with ERAS

\begin{tabular}{lccc}
\hline Variable & $\begin{array}{c}\text { Pre-ERAS } \\
(\mathrm{n}=161)\end{array}$ & $\begin{array}{c}\text { ERAS } \\
(\mathrm{n}=171)\end{array}$ & P-value \\
\hline Length of stay (day) & & & \\
$\quad$ Median (IQR) & $8.0(6.0-11.3)$ & $6.0(4.0-9.0)$ & $<0.0001$ \\
Mean \pm SD & $11.16 \pm 10.73$ & $8.56 \pm 10.22$ & 0.032 \\
Readmission within 30 days (\%) & 9.3 & 7.6 & 0.590 \\
Death within 30 days (\%) & 1.9 & 1.2 & 0.680 \\
\hline $\begin{array}{l}\text { ERAS, Enhanced Recovery After Surgery; IQR, interquartile range; SD, standard } \\
\text { deviation. }\end{array}$
\end{tabular}

the univariate linear and logistic regression models as tables, with the multivariate results reported in discussion and appendix rather than being formally tabled. We anticipate that our multivariate statistical model will improve as the prospectively collected database increases in size and in subsequent studies provide accurate multivariate modeling of ERAS interventions and LOS. The association between ERAS strategies that had a near $100 \%$ or $0 \%$ agreement rate and LOS (mainly preoperative) could not be estimated in this study. These included preadmission counseling, carbohydrate loading, immunonutrition, and oral antibiotics. There were missing data the NSQIP database collects different variable data for targeted colectomies and proctectomies, and when linked with our ERAS database which collects the same dataset for both colectomies and proctectomies, this resulted in missing data in various fields.

This study reported 7 high-yield ERAS interventions that influenced patient LOS the most-laparoscopic surgery, mobilization BD POD 0 to 1, discontinuation of IV fluids on POD 0 to 1, upgrading to solid diet by POD 0 to 2, removing the IDC by POD 0 to 2, avoiding NGT reinsertion, and removing drains early. Both major and minor complications increased LOS. Anastomotic leak and ileus were associated with the greatest increase in LOS.

\section{CONFLICT OF INTEREST}

Dr. Toh is the Chair of the ERAS Working Group, Agency for Clinical Innovation, Australia. No other potential conflict of interest relevant to this article was reported.

\section{ACKNOWLEDGMENTS}

The authors would like to thank Cancer Institute NSW (CINSW) for its Innovations in Cancer Control Variations in Care Grant (2018) (368646 RESX). The authors would also like to thank Sinan Albayati, Joris Harlaar, Jevon Puckett, Danette Wright, Gagandeep Bhullar, Carmen Hoffman, Ming Juan, Henry Wang, George Chen, Emma Hollands, Dinushi Perrera, Pratima Herle, Sara Shahrestani, Krystal Dinh, Carlin Ng, Kristian Adams, Ann Ho, Ben Kakala, Prasenjit Raychaudhuri, Lynleigh Evans, Paul 
Harnett, Arthur Richardson, and Gary Morgan.

\section{SUPPLEMENTARY MATERIALS}

Supplementary materials for this study are presented online (available at https://doi.org/10.3393/ac.2020.11.23).

\section{REFERENCES}

1. Kehlet H. Multimodal approach to control postoperative pathophysiology and rehabilitation. Br J Anaesth 1997;78:606-17.

2. Fearon KC, Ljungqvist O, Von Meyenfeldt M, Revhaug A, Dejong $\mathrm{CH}$, Lassen $\mathrm{K}$, et al. Enhanced recovery after surgery: a consensus review of clinical care for patients undergoing colonic resection. Clin Nutr 2005;24:466-77.

3. Ljungqvist O, Scott M, Fearon KC. Enhanced recovery after surgery: a review. JAMA Surg 2017;152:292-8.

4. Modasi A, Pace D, Godwin M, Smith C, Curtis B. NSAID administration post colorectal surgery increases anastomotic leak rate: systematic review/meta-analysis. Surg Endosc 2019;33:879-85.

5. Huang Y, Tang SR, Young CJ. Nonsteroidal anti-inflammatory drugs and anastomotic dehiscence after colorectal surgery: a meta-analysis. ANZ J Surg 2018;88:959-65.

6. Slim K, Joris J, Beloeil H; Groupe Francophone de Réhabilitation Améliorée après Chirurgie (GRACE). Colonic anastomoses and non-steroidal anti-inflammatory drugs. J Visc Surg 2016;153:26975.

7. Smith SA, Roberts DJ, Lipson ME, Buie WD, MacLean AR. Postoperative nonsteroidal anti-inflammatory drug use and intestinal anastomotic dehiscence: a systematic review and meta-analysis. Dis Colon Rectum 2016;59:1087-97.

8. Toh JW, Phan K, Hitos K, Pathma-Nathan N, El-Khoury T, Richardson AJ, et al. Association of mechanical bowel preparation and oral antibiotics before elective colorectal surgery with surgical site infection: a network meta-analysis. JAMA Netw Open 2018;1: e183226.

9. Chen M, Song X, Chen LZ, Lin ZD, Zhang XL. Comparing mechanical bowel preparation with both oral and systemic antibiotics versus mechanical bowel preparation and systemic antibiotics alone for the prevention of surgical site infection after elective colorectal surgery: a meta-analysis of randomized controlled clinical trials. Dis Colon Rectum 2016;59:70-8.

10. McSorley ST, Steele CW, McMahon AJ. Meta-analysis of oral antibiotics, in combination with preoperative intravenous antibiotics and mechanical bowel preparation the day before surgery, compared with intravenous antibiotics and mechanical bowel preparation alone to reduce surgical-site infections in elective colorectal surgery. BJS Open 2018;2:185-94.

11. Rollins KE, Javanmard-Emamghissi H, Acheson AG, Lobo DN. The role of oral antibiotic preparation in elective colorectal surgery: a meta-analysis. Ann Surg 2019;270:43-58.

12. Khuri SF. The NSQIP: a new frontier in surgery. Surgery 2005;
138:837-43.

13. Toh JW, Phan K, Ctercteko G, Pathma-Nathan N, El-Khoury T, Richardson A, et al. The role of mechanical bowel preparation and oral antibiotics for left-sided laparoscopic and open elective restorative colorectal surgery with and without faecal diversion. Int J Colorectal Dis 2018;33:1781-91.

14. Harris PA, Taylor R, Minor BL, Elliott V, Fernandez M, O’Neal L, et al. The REDCap consortium: building an international community of software platform partners. J Biomed Inform 2019;95: 103208

15. Harris PA, Taylor R, Thielke R, Payne J, Gonzalez N, Conde JG. Research electronic data capture (REDCap): a metadata-driven methodology and workflow process for providing translational research informatics support. J Biomed Inform 2009;42:377-81.

16. Clavien PA, Barkun J, de Oliveira ML, Vauthey JN, Dindo D, Schulick RD, et al. The Clavien-Dindo classification of surgical complications: five-year experience. Ann Surg 2009;250:187-96.

17. Clavien PA, Sanabria JR, Strasberg SM. Proposed classification of complications of surgery with examples of utility in cholecystectomy. Surgery 1992;111:518-26.

18. Heriot A, Platell C, Byrne C, Chapuis P, Doudle M, McMurrick P, et al. The 2017 Binational Colorectal Cancer Audit report. Hawthorn, VIC: Binational Colorectal Cancer Audit; 2017. 58 p.

19. Iversen H, Ahlberg M, Lindqvist M, Buchli C. Changes in clinical practice reduce the rate of anastomotic leakage after colorectal resections. World J Surg 2018;42:2234-41.

20. Lluis N, Biondo S. Prolonged postoperative ileus after colorectal surgery: still an unresolved problem. Ann Laparosc Endosc Surg 2018;3:15.

21. Hewett PJ, Allardyce RA, Bagshaw PF, Frampton CM, Frizelle FA, Rieger NA, et al. Short-term outcomes of the Australasian randomized clinical study comparing laparoscopic and conventional open surgical treatments for colon cancer: the ALCCaS trial. Ann Surg 2008;248:728-38.

22. Guillou PJ, Quirke P, Thorpe H, Walker J, Jayne DG, Smith AM, et al. Short-term endpoints of conventional versus laparoscopicassisted surgery in patients with colorectal cancer (MRC CLASICC trial): multicentre, randomised controlled trial. Lancet 2005; 365:1718-26.

23. Daams F, Luyer M, Lange JF. Colorectal anastomotic leakage: aspects of prevention, detection and treatment. World J Gastroenterol 2013;19:2293-7.

24. Stevenson AR, Solomon MJ, Lumley JW, Hewett P, Clouston AD, Gebski VJ, et al. Effect of laparoscopic-assisted resection vs open resection on pathological outcomes in rectal cancer: the $\mathrm{ALaC}$ aRT randomized clinical trial. JAMA 2015;314:1356-63.

25. Ngui NK, Hitos K, Ctercteko G. Preoperative factors prolonging the length of stay in elective colorectal surgery. ANZ J Surg 2011; 81:624-8.

26. Ahmed J, Lim M, Khan S, McNaught C, Macfie J. Predictors of length of stay in patients having elective colorectal surgery within an enhanced recovery protocol. Int J Surg 2010;8:628-32. 


\section{Coloproctology James Wei Tatt Toh, et al.}

27. Boulind CE, Yeo M, Burkill C, Witt A, James E, Ewings P, et al. Factors predicting deviation from an enhanced recovery programme and delayed discharge after laparoscopic colorectal surgery. Colorectal Dis 2012;14:e103-10.

28. Smart NJ, White P, Allison AS, Ockrim JB, Kennedy RH, Francis NK. Deviation and failure of enhanced recovery after surgery following laparoscopic colorectal surgery: early prediction model. Colorectal Dis 2012;14:e727-34.

29. Ahmed J, Khan S, Lim M, Chandrasekaran TV, MacFie J. Enhanced recovery after surgery protocols: compliance and variations in practice during routine colorectal surgery. Colorectal Dis 2012;14:1045-51.

30. Roulin D, Muradbegovic M, Addor V, Blanc C, Demartines N, Hübner M. Enhanced recovery after elective colorectal surgery: reasons for non-compliance with the protocol. Dig Surg 2017;34: 220-6.
31. Lovely JK, Maxson PM, Jacob AK, Cima RR, Horlocker TT, Hebl JR, et al. Case-matched series of enhanced versus standard recovery pathway in minimally invasive colorectal surgery. Br J Surg 2012;99:120-6.

32. Geltzeiler CB, Rotramel A, Wilson C, Deng L, Whiteford MH, Frankhouse J. Prospective study of colorectal enhanced recovery after surgery in a community hospital. JAMA Surg 2014;149:95561.

33. Miller TE, Thacker JK, White WD, Mantyh C, Migaly J, Jin J, et al. Reduced length of hospital stay in colorectal surgery after implementation of an enhanced recovery protocol. Anesth Analg 2014;118:1052-61.

34. Thiele RH, Rea KM, Turrentine FE, Friel CM, Hassinger TE, McMurry TL, et al. Standardization of care: impact of an enhanced recovery protocol on length of stay, complications, and direct costs after colorectal surgery. J Am Coll Surg 2015;220:430-43. 
Volume 38, Number 1, 2022

Ann Coloproctol 2022;38(1):36-46

Supplementary Table 1. Univariate linear and logistic regression of patient, disease and surgical variables and hospital length of stay (LOS)

\begin{tabular}{|c|c|c|c|c|}
\hline Patient variable & $\begin{array}{l}\text { Univariate linear regression } \\
\text { LOS }(95 \% \text { CI) (day) }\end{array}$ & $\mathrm{P}$-value & $\begin{array}{l}\text { Univariate logistic regression for } \\
\text { LOS of } \leq 6 \text { vs. }>6 \text { days } 0 \mathrm{R}(95 \% \mathrm{Cl})\end{array}$ & P-value \\
\hline Male sex & $8.4(6.4-10.5)$ vs. $8.8(3.6-14.0)$ & 0.819 & 2.048 (1.097-3.825) & 0.024 \\
\hline Age & B 0.108 (S.E. 0.063) & 0.093 & No estimate & \\
\hline \multicolumn{5}{|l|}{ ASA PS classification } \\
\hline I & $7.4(1.4-13.4)$ & 0.023 & Reference & 0.267 \\
\hline$\|$ & $6.6(5.6-7.5)$ & & $1.755(0.512-6.021)$ & 0.371 \\
\hline III & $11.2(7.8-14.6)$ & & $2.521(0.731-8.694)$ & 0.143 \\
\hline BMI & No estimate & & $0.987(0.930-1.047)$ & 0.659 \\
\hline $\mathrm{HbA1c}$ & No estimate & & $1.076(0.734-1.579)$ & 0.707 \\
\hline \multicolumn{5}{|l|}{ Disease variable } \\
\hline Cancer & $8.8(7-10.5)$ vs. $7.8(4.3-11.3)$ & 0.619 & $1.261(0.571-2.782)$ & 0.566 \\
\hline Crohn disease & $5.5(0-37.3)$ vs. $8.6(7.0-10.2)$ & 0.671 & $1.386(0.085-22.534)$ & 0.819 \\
\hline Ulcerative colitis & No estimate & No estimate & No estimate & No estimate \\
\hline Diverticulitis & 7.7 (5.2-10.2) vs. 8.6 (7.0-10.3) & 0.751 & $1.200(0.385-3.737)$ & 0.753 \\
\hline Volvulus & No estimate & No estimate & No estimate & No estimate \\
\hline Others & $9.2(1.3-17.2)$ vs. $8.5(6.9-10.1)$ & 0.804 & $0.749(0.240-2.339)$ & 0.619 \\
\hline \multicolumn{5}{|l|}{ Surgical variable } \\
\hline Surgical access & $10.8(6.3-15.26)$ & 0.0697 & Reference & 0.024 \\
\hline Open & $7.0(5.1-9.0)$ & & $0.51(0.19-1.33)$ & 0.167 \\
\hline Laparoscopic & $10.6(6.0-15.2)$ & & $2.17(0.59-7.99)$ & 0.246 \\
\hline Conversion to open & $12.2(8.1-16.4)$ & & $1.09(0.33-3.62)$ & 0.887 \\
\hline \multicolumn{5}{|l|}{ Hybrid } \\
\hline \multicolumn{5}{|l|}{ Type of procedure } \\
\hline Right & $6.7(5.4-7.9)$ & 0.419 & Reference & 0.098 \\
\hline Left & 6.5 (3.5-9.6) & & $1.714(0.225-13.086)$ & 0.603 \\
\hline High anterior resection & $9.5(4.6-14.5)$ & & $0.719(0.310-1.669)$ & 0.442 \\
\hline Low anterior resection & $11.0(7.7-14.3)$ & & 2.743 (1.184-6.354) & 0.019 \\
\hline Unspecified & $8.7(4.7-12.7)$ & & $1.286(0.262-6.310)$ & 0.757 \\
\hline Other & $8.6(7-10.1)$ & & $1.371(0.468-4.015)$ & 0.564 \\
\hline
\end{tabular}

ASA, American Society of Anesthesiologist; PS, physical status; HbA1c, glycosylated haemoglobin A1c; BMl, body mass index; LOS, length of stay. 


\section{Coloproctology}

James Wei Tatt Toh, et al.

Supplementary Table 2. Patient demographics, indications for surgery and procedural characteristics

\begin{tabular}{|c|c|c|}
\hline Patient characteristics & $\%^{a}$ & Data not available (n) \\
\hline $\mathrm{Age}^{\mathrm{b}}$ & $66.7(57.6-74.9)$ & \\
\hline Female sex & 42.1 & \\
\hline ASA PS classification & & 8 \\
\hline I & 8.8 & \\
\hline$\|$ & 45.6 & \\
\hline III & 40.9 & \\
\hline Body mass index & $27.8(24.2-31.7)$ & \\
\hline $\mathrm{HbA1} \mathrm{c}^{\mathrm{b}}$ & $5.6(5.3-6.3)$ & \\
\hline Preadmission counselling & 100 & 1 \\
\hline Carbohydrate loading & 97.1 & 1 \\
\hline Immunonutrition & 100 & 6 \\
\hline Indication for surgery & & 0 \\
\hline Cancer & 81.3 & \\
\hline Crohn disease & 1.2 & \\
\hline Ulcerative colitis & 0.5 & \\
\hline Diverticulitis & 7.6 & \\
\hline Recurrent volvulus & 0 & \\
\hline Others & 8.2 & \\
\hline Procedure & 0 & \\
\hline Right hemicolectomy & 33.3 & \\
\hline Transverse colectomy & 0.6 & \\
\hline Left hemicolectomy & 2.3 & \\
\hline High anterior resection & 26.3 & \\
\hline Low anterior resection & 12.3 & \\
\hline Ultralow anterior resection & 10.5 & \\
\hline Anterior resection unspecified & 4.1 & \\
\hline Hartmann's procedure & 1.8 & \\
\hline Abdominoperineal resection & 0.6 & \\
\hline Total/Subtotal colectomy & 1.2 & \\
\hline Total proctocolectomy \pm IPAA & 1.8 & \\
\hline Others & 5.3 & \\
\hline
\end{tabular}

ASA, American Society of Anesthesiologist; PS, physical status; HbA1c, glycosylated haemoglobin A1c; IPAA, ileal pouch anal anastomosis.

aDoes not include missing data. ${ }^{\mathrm{b}}$ Median (interquartile range). 
Supplementary Table 3. Compliance with ERAS interventions

\begin{tabular}{|c|c|c|}
\hline ERAS intervention & $\begin{array}{l}\text { Agreement } \\
\text { rate }(\%)^{\mathrm{a}}\end{array}$ & $\begin{array}{c}\text { Data not } \\
\text { available (n) }\end{array}$ \\
\hline Preadmission counselling & 100 & 1 \\
\hline Carbohydrate loading & 97.1 & 1 \\
\hline Immunonutrition & 0 & 11 \\
\hline Surgical access & & 0 \\
\hline Open & 11.7 & \\
\hline Laparoscopic & 63.2 & \\
\hline Laparoscopic converted to open & 11.1 & \\
\hline Hybrid & 14.0 & \\
\hline Bowel preparation & 91.2 & 0 \\
\hline Oral antibiotics & 1.2 & 0 \\
\hline Liquids up to $3 \mathrm{hr}$ prior to surgery & 73.7 & 0 \\
\hline Epidural & 5.3 & 0 \\
\hline Multimodal pain management & 88.2 & 1 \\
\hline NSAIDs & 31.8 & 1 \\
\hline Preoperative iron transfusion for anemia & 6.3 & 12 \\
\hline Postoperative antiemetics & 85.4 & 0 \\
\hline Mobilization POD 0-1 & 83.0 & 0 \\
\hline Mobilization BD POD 0-1 & 82.9 & 19 \\
\hline IV fluids discontinued POD 0-1 & 40.1 & 4 \\
\hline Upgraded to solid diet POD 0-2 & 71.4 & 0 \\
\hline \multicolumn{3}{|l|}{ IDC } \\
\hline IDC out POD 0-2 & 79.5 & 0 \\
\hline Failed TOV, IDC reinsertion required & 8.8 & 0 \\
\hline \multicolumn{3}{|l|}{ NGT } \\
\hline NGT out POD 0-2 & 3.5 & \\
\hline NGT out $P O D \geq 3$ & 8.8 & \\
\hline No NGT & 87.7 & \\
\hline NGT reinsertion & 14.6 & 0 \\
\hline Drain & 1.0 & \\
\hline No drain & 54.7 & \\
\hline Drain removed POD 0-2 & 10.6 & \\
\hline Drain removed POD 3-4 & 17.6 & \\
\hline Drain removed POD $\geq 5$ & 14.6 & 0 \\
\hline
\end{tabular}

ERAS, Enhanced Recovery After Surgery; NSAIDs, nonsteroidal anti-inflammatory drugs; POD, postoperative day; BD, twice a day; IV, intravenous; IDC, indwelling catheter; TOV, trial of void; NGT, nasogastric tube.

${ }^{a}$ Does not include missing data. 
The impact of variations in care and complications within a colorectal Enhanced Recovery After Surgery program on length of stay

\section{Coloproctology James Wei Tatt Toh, et al.}

\section{Bowel preparation}

NOT ROUTINE for right side.

Left-sided resections: bowel preparation day before surgery with glycoprep (PEG)

or sodium phosphate prep

\section{DVT prophylaxis}

TED stockings; clexane $20 \mathrm{mg}$ daily for low-risk patients, $40 \mathrm{mg}$ daily for highrisk patients

\section{Oral analgesia}

Paracetamol $1 \mathrm{~g}$ QID

Oxycodone

$<50$ yr: $5-10 \mathrm{mg} 2-4 \mathrm{hr}$ PRN $50-70$ yr: $5 \mathrm{mg} \mathrm{2}-4 \mathrm{hr}$ PRN $>70$ yr: $2.5 \mathrm{mg} \mathrm{2}-4 \mathrm{hr}$ PRN

COX-II inhibitor once oral intake re-established

\section{Discharge orders}

Oxycodone (Endone)

$<50$ yr: $5-10 \mathrm{mg} 2-4 \mathrm{hr}$ PRN 50-70 yr: $5 \mathrm{mg} 2-4 \mathrm{hr}$ PRN $>70$ yr: $2.5 \mathrm{mg} 2-4 \mathrm{hr}$ PRN

Paracetamol $1 \mathrm{~g}$ QID Follow-up appointment made Discharge summary completed

\section{Preoperative}

ERAS education; Discharge planning; incentive spirometry

Carbohydate loading: 4 cartons of Nutricia Preop ${ }^{\mathrm{TM}}$ evening before surgery, 2 cartons $3 \mathrm{hr}$ prior to surgery, unless contra-indicated
Intraoperatively

Normothermia with bair-hugger Regional field block for open surgery. DVT prophylaxis.

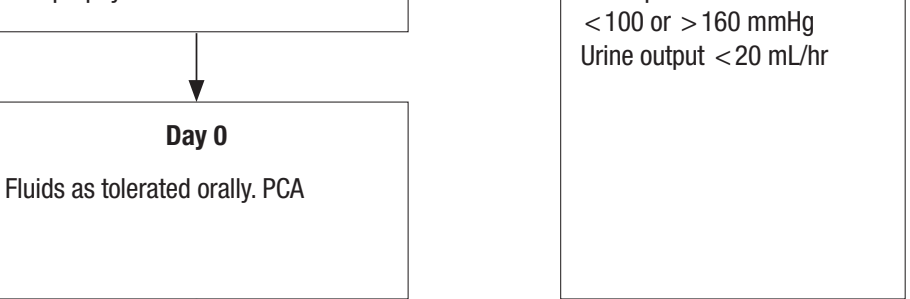

\section{Notify RMO/PACE call}

Temperature $>38^{\circ} \mathrm{C}$

Pulse $>120$ beats/min

Blood pressure

$<100$ or $>160 \mathrm{mmHg}$

Urine output $<20 \mathrm{~mL} / \mathrm{h}$

\section{Day 1}

Full blood count/electrolytes/glucose/creatinine Free fluids as tolerated, regular ensure

$2 \mathrm{~L}$ of $0.18 \mathrm{NaCl}, 4 \%$ dextrose IV IDC out Mobilize to $80 \mathrm{~m}$

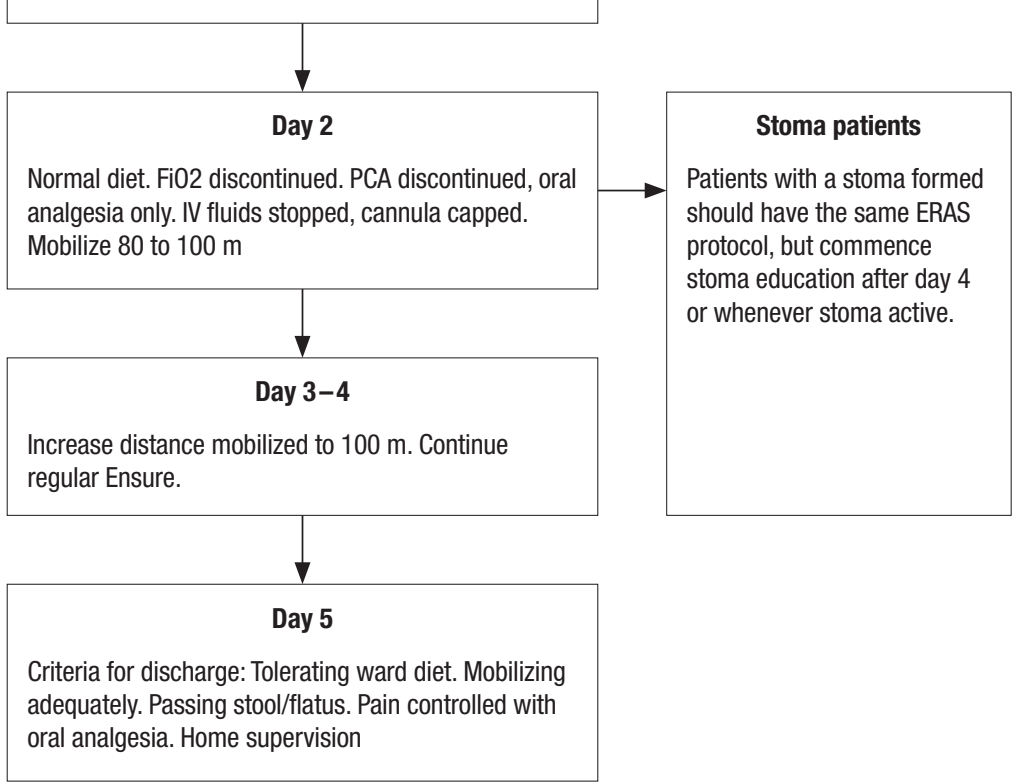

Supplementary Fig. 1. Westmead ERAS (2017-2018). ERAS, Enhanced Recovery After Surgery; TED, thrombo-embolus deterrent; PEG, polyethylene glycol; QID, quater in die (4 times each day); PRN, pro re nata (as needed); COX-II, cyclo-oxygenase-2; DVT, deep venous thrombosis; IV, intravenous; IDC, indwelling catheter; FiO2, fraction of inspired oxygen; PCA, patient controlled analgesia; RMO, resident medical officer; PACE, patient with acute condition for escalation. 


\begin{tabular}{|l|}
\multicolumn{1}{|c|}{$\begin{array}{c}\text { Bowel preparation/oral } \\
\text { antibiotics }\end{array}$} \\
$\begin{array}{l}\text { All patients undergoing elective colorectal } \\
\text { surgery suitable should be prescribed: }\end{array}$ \\
$1 \mathrm{~g} \mathrm{neomycin}+400 \mathrm{mg}$ metronidazole at \\
$7 \mathrm{am}, 3 \mathrm{pm}, 10 \mathrm{pm}$ the day before surgery \\
with glycoprep (PEG) or picoprep (sodium \\
phosphate) $11,2,5 \mathrm{pm}$.
\end{tabular}

\begin{tabular}{l} 
DVT prophylaxis \\
TED stockings; clexane $20 \mathrm{mg}$ daily for low- \\
risk \\
patients, $40 \mathrm{mg}$ daily for high-risk patients. \\
Cancer patients may benefit from $20 \mathrm{mg}$ \\
clexane daily SC for $2-4$ wks post discharge \\
\hline
\end{tabular}

Oral analgesia (day 2-)
Multimodal
As per pain team (see separate pain protocol)
Avoid non-selective NSAIDs
Selective NSAIDs as per surgeon/pain team

Discharge orders
Paracetamol $1 \mathrm{~g}$ qid 5 days
\pm Selective NSAIDs
Oxycodone
$<70 \mathrm{yr}: 5 \mathrm{mg} / 4 \mathrm{hr}$ PRN
$>70 \mathrm{yr}: 2.5 \mathrm{mg} / 4 \mathrm{hr}$ PRN
Follow-up appointment made
Cancer patients should be referred to MDT
for discussion
Cancer patients may benefit from $20 \mathrm{mg}$
clexane SC daily for $2-4$ wks

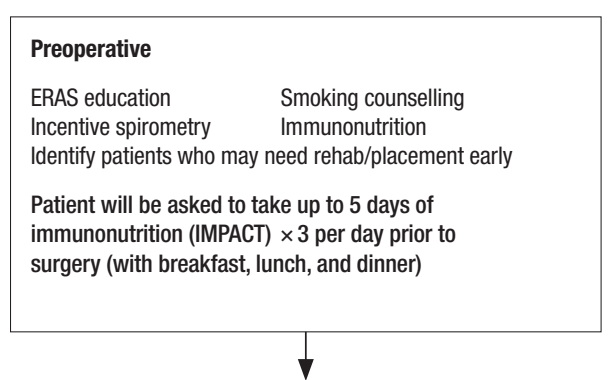

\section{Intraoperatively}

Clexane $20 \mathrm{mg}$ or heparin 5,000 U SC

IV antibiotics at induction

Normothermia with bair-hugger

Betadiene/Chlorhexidine \pm alcohol preparation

Wound protectors, closing tray, change of gloves prior to closure

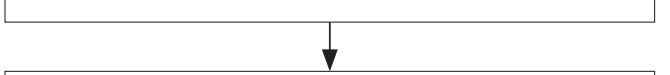

\section{Day 0}

Remove NGT

Free fluids as tolerated orally. PCA. Regular Maxolon and PRN Ondansetron IV $48 \mathrm{hr}$, then oral for 3 days

\section{1}

Day 1

$\mathrm{FBC/UEC/check} \mathrm{baseline} \mathrm{BSLs} \mathrm{(+} \mathrm{diabetes} \mathrm{mx}$ )

Free fluids as tolerated, regular Ensure

$4 \%$ dextrose and $1 / 5$ normal saline per $12 \mathrm{hr}$

Continue oxygen supplementation (FiO2) nasal prongs

IDC out. For proctectomies, IDC may be removed D2-3

Mobilize to at least $80 \mathrm{~m}$ ( \pm physiotherapy) bd

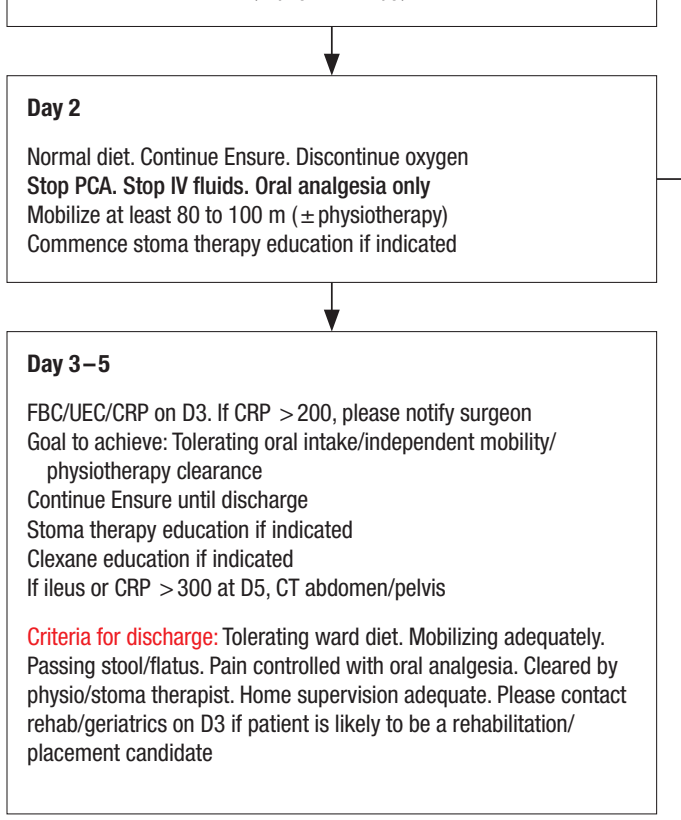

\begin{tabular}{|lr|}
\hline \multicolumn{2}{|l|}{ Preoperative patient instructions: } \\
$7 \mathrm{am}$ & Duration \\
$1 \mathrm{~g}$ neomycin & 1 day \\
$400 \mathrm{mg}$ metronidazole & 1 day \\
& \\
$3 \mathrm{pm}$ & 1 day \\
$1 \mathrm{~g} \mathrm{neomycin}$ & 1 day \\
$400 \mathrm{mg}$ metronidazole & \\
& \\
$10 \mathrm{pm}$ & 1 day \\
$1 \mathrm{~g} \mathrm{neomycin}$ & 1 day \\
400 mg metronidazole &
\end{tabular}

On day before surgery, take your bowel preparation at $11 \mathrm{am}, 2 \mathrm{pm}$, and $5 \mathrm{pm}$

IMPACT drink with breakfast lunch and dinner for up to 5 days before surgery

\section{Notify RMO/PACE call}

Temperature $>38^{\circ} \mathrm{C}$

Pulse $>120$ beats $/ \mathrm{min}$

Blood pressure $<100$ or $>160 \mathrm{mmHg}$ Urine outcome $<15 \mathrm{~mL} / \mathrm{hr}$

\section{Ileus}

If patient is not tolerating oral intake/ vomiting/abdominal distension D2-5, then notify treating surgeon and may need to continue IV fluids, SC morphine for analgesia and perform appropriate investigation.

Ensure all patients are on regular Maxolon and PRN Ondansetron

Optional: chewing gum

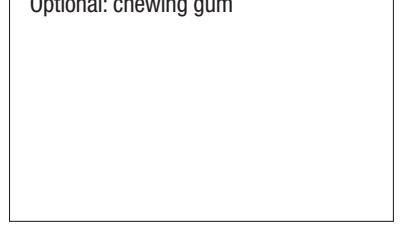

\section{Drain}

No drain for right sided colectomy

For left sided colectomy or proctectomy, please follow appropriate guidance from surgeon in charge

Supplementary Fig. 2. Westmead ERAS (2019-). ERAS, Enhanced Recovery After Surgery; PEG, polyethylene glycol; DVT, deep venous thrombosis; TED, thromboembolus deterrent; NSAIDs, non-steroidal anti-inflammatory drugs; MDT, multidisciplinary team; SC, subcutaneous; qid, quarter in die (4 times each day); PCA, patient controlled analgesia; NGT, nasogastric tube; PRN, pro re nata (as needed); IV, intravenous; FBC, full blood count; UEC, urea electrolytes and creatinine; BSLs, blood sugar levels; IDC, indwelling catheter; FiO2, fraction of inspired oxygen; bd, bis die (twice a day); CRP, C-reactive protein; D, day; CT, computed tomography. 